\title{
Escala de Clima Escolar: adaptación al español y validación en estudiantes chilenos*
}

\author{
School Climate Scale: Spanish Adaptation \\ and Validation in Chilean Students
}

Recibido: marzo 15 de 2013 | Revisado: febrero 27 de 2014 | Aceptado: febrero 27 de 2014

\author{
VERÓNICA LÓPEZ** \\ M. ÁNGELES BILBAO *** \\ PAULA ASCORRA \\ IVÁN MOYA DiEZ \\ MACARENA MORALES \\ Pontificia Universidad Católica de Valparaíso, Chile
}

doi:10.11144/Javeriana.UPSY13-3.ecea

Para citar este artículo: López, V., Bilbao, M. Á., Ascorra, P., Moya, I., \& Morales, M. (2014). Escala de Clima Escolar: adaptación al español y validación en estudiantes chilenos. Universitas Psychologica, 13(3), 1111-1122. http://dx.doi.org/10.11144/Javeriana.UPSY13-3.ecea

" Este estudio fue financiado por los Proyectos FONDECYT 1110859 y FONDEF CA12I10243

* Profesora Adjunta, Escuela de Psicología, Pontificia Universidad Católica de Valparaíso. Investigadora Asociada, Centro de Investigación Avanzada en Educación. Correo electrónico: veronica.lopezl@ gmail.com

**** Escuela de Psicología. Correos electrónicos: bilbao. angeles@gmail.com, pascorra@ucv.cl, ivanmd@ gmail.com, macarena.psi@hotmail.com

\section{RES U MEN}

El clima escolar resulta de creciente interés para investigadores e instituciones educativas. Las estrategias de mejoramiento escolar basadas en evidencias han incluido el clima escolar para apoyar a los integrantes de comunidades educativas en la creación de escuelas seguras y comprometidas con el aprendizaje. Es necesario contar con instrumentos validados para la población latinoamericana, que permitan la investigación comparada. Presentamos los resultados de la validación de la Escala de Clima Escolar. El instrumento mide la percepción de estudiantes sobre la aplicación de normas, el apoyo de adultos y la participación. Se tradujo al español y se realizó un análisis psicométrico. La muestra fue de 4688 estudiantes chilenos, de 4ㅜㅜ $6^{\circ}$ y $8^{\circ}$ básico. Los resultados evidencian un alto nivel de consisten interna $(\alpha=0.89)$ y de varianza explicada (54\%). El modelo de tres factores mostró un buen ajuste, así como uno de cuatro factores, que separa las normas generales de las normas contra la violencia sexual.

Palabras clave

clima escolar; escala; validación; fiabilidad; validez

\section{A B S T R A C T}

School climate has received increased attention use by researchers and educational institutions. Evidence-based school improvement processes have included school climate as a means of helping create schools that are safe and committed with learning. It is necessary to count with valid measures of school climate for Latin American countries, which may allow for comparative research. We present the results of the validation of the School Climate Scale. The instrument measures students' perceptions of norms, support from adults in the school, and participation. The scale was translated into Spanish and its psychometric properties were studied on a sample of 4,688 Chilean students from grades fourth, sixth, and eight. Findings show a high level on internal consistency $(\alpha=0.89)$ and explained variance $(54 \%)$. Structural equation models fit the data well, both for the three-factor solution as well a four-factor structure that separates general school norms from norms against sexual violence. The scale can facilitate data-driven decisions and may be incorporated into evidenced-based school improvement processes.

Keywords

school climate; scale; validation; reliability; validity 
En los últimos años, los estudios de la calidad educativa han destacado entre sus factores al contexto escolar como elemento de relevancia. Entre los elementos analizados en este nivel, el clima escolar ha resultado de creciente interés y uso por parte de agencias, ministerios e instituciones educativas de envergadura internacional (Thapa, Cohen, Guffey \& Higgins-D’Alessandro, 2013). En países como Estados Unidos, Francia, Canadá e Israel, el clima escolar forma parte de la evaluación de calidad educativa. Así también, las estrategias de mejoramiento escolar basadas en evidencias han incluido el clima escolar para apoyar a los estudiantes, al personal y a los padres y apoderados en la generación de escuelas más seguras, apoyadoras y comprometidas con el aprendizaje.

En estos países, cada vez se hace más necesario contar con instrumentos psicométricamente sólidos que permitan a los investigadores y a las instituciones educativas incorporar la medición del clima escolar dentro de los procesos de mejoramiento escolar basados en evidencias (Zulllig, Collins, Ghani, Patton, Huebner, \& Ajemie, 2014). Sin embargo, en los países de habla hispana y en América Latina, son pocos los instrumentos disponibles cuyas características psicométricas se hayan estudiado y difundido (Aron, Milicic \& Armijo, 2012; Trianes, Blanca, de la Morena, Infante \& Raya, 2006). Por otra parte, es necesario contar con instrumentos validados para la población latinoamericana, que permitan la investigación comparada entre los países de la Región, así como con países de otros continentes. Desde una perspectiva social-ecológica (Benbenishty \& Astor, 2005; Bronfenbrenner \& Morris, 2006; Swearer et al., 2012), los resultados de aprendizaje de los estudiantes debieran considerar la interrelación entre los distintos niveles que operan en y a través del sistema escolar. Variables contextuales como el clima de aula y el clima escolar han mostrado relaciones positivas con el rendimiento escolar (Brand, Felner, Seitsinger \& Hupkau, 2006; Lopez \& Ascorra, 2012; López, Ascorra et al., 2012 Nim., van Damme, Liu, Vanlaar \& Gielen, 2013). Por el contrario, altos niveles de victimización y violencia escolar se asocian negativamente al clima de aula (López, Bilbao \& Rodríguez, 2012) y clima escolar (Marshall, 2005).

Desde la perspectiva social-ecológica (Bronfenbrenner \& Morris, 2006; Khoury-Kassabri, Benbenishty, Astor \& Zeira, 2004), la violencia escolar es un fenómeno en el cual se conjugan elementos desde múltiples niveles de análisis, entre ellos, los niveles individuales, diádicos, grupales, sociales y culturales Incluso algunos autores proponen considerar el no respeto a la etnia de los estudiantes como un tipo de violencia simbólica (Jimenez, 2012). No obstante, la investigación sobre violencia escolar ha tendido a focalizarse en el bullying o victimización entre pares, sin considerar los espacios de relación que se crean en el aula y en la escuela y que inciden en las conductas de victimización. De allí, se hace necesario contribuir desde la investigación empírica a la visibilización de los factores sociales y afectivos implicados en el clima de aula y en el clima escolar que impactan en los niveles de violencia escolar y en el bienestar de los estudiantes (Véliz-Burgos, 2012). Para esto, es necesario contar con instrumentos validados y que puedan entrar en diálogo con la investigación en el concierto internacional. En Chile, por ejemplo, si bien ha habido esfuerzos por incorporar medidas de clima escolar en las últimas encuestas nacionales, la falta de ítems comparables con otros países dificulta la investigación transcultural.

En este estudio, se presentan los resultados de la validación de un instrumento empleado a nivel internacional, la Escala de Clima Escolar (Benbenishty \& Astor, 2005), aplicada a una muestra representativa de estudiantes de enseñanza básica de la región de Valparaíso.

\section{El clima escolar en clave histórica}

Si bien las organizaciones educacionales son un tipo más de sistemas organizacionales, algunos autores como Orton y Weick (1990) postulan que los sistemas educacionales -las escuelas, los liceos, los institutos de enseñanza- poseen características muy distintas de los sistemas organizacionales productivos -como las empresas manufactureras y de servicios-. 
Dado que las organizaciones educacionales son sistemas débilmente estructurados (Orton \& Weick, 1990), es decir, organizaciones cuya riqueza organizativa se articula en los espacios de informalidad, construyendo desde allí un entramado de relaciones de poder que operan paralelas a la línea jerárquica formal de la organización, parece poco pertinente evaluar el clima educacional, utilizando las dimensiones comúnmente empleadas para evaluar clima organizacional.

El clima escolar (CE), por lo tanto, se plantea como una adaptación del constructo de clima organizacional (CO) a establecimientos educacionales. Se conservan en el CE todas las características del $\mathrm{CO}$, es decir, la concepción de una representación cognitiva dinámica, pero relativamente estable en el tiempo y que otorga la posibilidad de distinguir el sistema educacional de otro tipo de sistemas (Ascorra, Aras \& Graf, 2003). Esta representación estaría conformada por las percepciones de los miembros que estudian en una organización en relación con una serie de variables educacionales.

Los primeros instrumentos orientados a medir $\mathrm{CE}$, como un constructo distinto al de $\mathrm{CO}$, aparecen en la década de los 80 , y se construyen funda- mentalmente como medidas de satisfacción con la escuela y sentido de pertenencia a ella. Tal es el caso del School Climate Survey Form (Kelley, 1989) y de la Escala del Clima Social de Moos (Moos, Moos \& Tricket, 1987; Moos \& Trickett, 1974).

Las escalas más recientes utilizadas a nivel internacional tienden a medir el CE desde concepciones más abiertas, pero también más heteroglósicas. Por ejemplo, el California School Climate Survey (Furlong, 2005) y la School Climate Scale (Haynes, Emmons \& Comer, 1993) contienen dimensiones que miden motivación de logro, justicia, orden y disciplina, implicación de los padres, recursos compartidos, relaciones interpersonales de los estudiantes y relaciones estudiantes-profesor.

\section{Hacia una definición de clima escolar}

Como hemos visto, resulta difícil hablar del clima de la escuela como un constructo unidimensional y referido a un solo nivel de análisis. Desde una perspectiva social-ecológica, sería necesario distinguir, a lo menos, dos niveles de climas en la escuela: un micro-clima de aula y un meso-clima de escuela.

TABLA 1

Dimensiones implicadas en la evaluación del clima escolar

\begin{tabular}{lll}
\hline Dimensión & Autores & $\begin{array}{l}\text { Instrumentos que } \\
\text { evalúan esta dimensión }\end{array}$ \\
\hline \multirow{2}{*}{ Normas justas } & (Benbenishty \& Astor, 2005; Cornejo \& Redondo, 2001; Furlong, & CSCSS; CHKS; SES; \\
& 2005; Guerra, Castro \& Vargas, 2011; Marjoribanks, 1980). & SCI \\
\hline & $\begin{array}{l}\text { (Aron, Milicic \& Armijo, 2012; Benbenishty \& Astor, 2005; Cornejo } \\
\text { \& Redondo, 2001; Furlong, 2005; Furlong \& Morrison, 1995; INEE, }\end{array}$ & $\begin{array}{l}\text { CSCSS; CHKS; } \\
\text { INEE; SES; SCI; }\end{array}$ \\
Apoyo de profesores & $\begin{array}{l}\text { 2008; Marjoribanks, 1980; Laboratorio Latinoamericano de la } \\
\text { Calidad de la Educación [LLECE], 2008; Trianes, Blanca, Morena, }\end{array}$ & $\begin{array}{l}\text { SERCE; CECSCE; } \\
\text { Infante \& Raya, 2006; Werblow, Robinson \& Duesbery, 2010). }\end{array}$ \\
\hline Participación & (Benbenishty \& Astor, 2005). & SCI \\
\hline $\begin{array}{l}\text { Pertenencia y } \\
\text { conexión }\end{array}$ & (Cornejo \& Redondo, 2001; Guerra et al., 2011; Goodenow, 1993; & CHKS; SES; SERCE \\
\hline $\begin{array}{l}\text { Orientación al } \\
\text { aprendizaje }\end{array}$ & (Corjoribanks, 1980; LLECE, 2008). & \\
\hline Seguridad & LLECE, 2008; Werblow, Robinson \& Duesbery, 2010). & SES; SERCE \\
\hline
\end{tabular}

Nota . CSCSS $=$ California School Climate and Safety Survey (Furlong et al., 2005); CHKS = California Healthy Kids Survey; BIECE = Batería de Instrumentos para la Evaluación del Clima Escolar (INEE, 2008); SES = School Environment Scale (Marjoribanks, 1980, adaptado por Cornejo \& Redondo, 2001) SCI = School Climate Inventory (Benbenishty \& Astor, 2005); SERCE = Segundo Estudio Regional Comparativo y Explicativo (LLECE, 2008); CECSCE = Cuestionario de Clima Social del Centro Escolar (Trianes et al., 2006); ECLIS = Escala de Clima Social Escolar (Aron et al., 2012)

Fuente: elaboración propia 
En el nivel meso, del cual se ocupa el presente artículo, se puede situar al Clima Escolar (CE). Assael y Neumann (1991) definen el CE como un constructo multidimensional que hace referencia a las percepciones, pensamientos y valores que los miembros de un establecimiento educacional construyen de este y de las relaciones que en él se dan. Se entiende que el CE es una característica de los establecimientos educacionales producida por las percepciones de los alumnos respecto a variables internas, que a su vez, produce percepciones particulares. Es decir, la modalidad, tipo y profundidad de las interacciones sociales y los comportamientos individuales generan determinado clima; el cual, a su vez, repercute en la producción de determinadas modalidades, profundidad y frecuencia de relaciones e interacciones sociales y comportamientos individuales.

La característica multidimensional del CE plantea desafíos para su evaluación. Tras una revisión de las dimensiones implicadas en los principales instrumentos de evaluación, López el al. (2012) concluyeron que estos, en general, buscan determinar el CE desde las percepciones que manifiestan los estudiantes sobre de la aplicación consistente de normas y sobre el trato que se genere entre pares y entre alumnos y profesores (ver Tabla 1).

Sin embargo, no solo es importante la percepción de buen trato, o al revés, una percepción de buen trato no garantiza un clima escolar favorable y propicio para el aprendizaje. También hay elementos de la gestión escolar que inciden significativamente en la generación y permanencia de ambientes de aprendizaje favorables para el aprendizaje y para el bienestar de la comunidad. Como señalan Mena, Becerra y Castro (2011), la buena convivencia no ocurre "espontáneamente" sino que debe gestionarse activa y democráticamente.

La percepción de autonomía o agenciamiento en los procesos de toma de decisiones está asociado a una mejor percepción del CE (Johnson, 2010). Por otra parte, un estilo de liderazgo autoritario tiende a instalar prácticas de castigo y aplicación arbitraria de normas, lo que incide negativamente en la percepción que tienen los miembros de la comunidad escolar respecto del cumplimiento de las normas y del trato justo (Carrasco, López y Estay, 2012).
Tomando estos elementos en consideración, Benbenishty y Astor (2005) han especificado tres elementos implicados en la generación de un CE favorable:

a) Normas y políticas claras (policy). Como se ha visto, la literatura indica que las escuelas con políticas claras y consistentes para abordar la violencia escolar han sido capaces de reducir los niveles de violencia en comparación a otras escuelas sin estas políticas. Es necesario que estas políticas sean percibidas como justas por los miembros de la comunidad, es decir, aplicadas con sentido de justicia y no con arbitrariedad (Johnson, 2010; Milicic \& Aron, 2000).

b) Relaciones positivas y de apoyo con adultos (supportive positive relationships with adults). $\mathrm{El}$ apoyo que los estudiantes reciban de parte de los profesores y de los otros adultos en la escuela incide positivamente en el bienestar subjetivo y social de los estudiantes, en la medida en que las relaciones positivas favorecen la confianza y compromiso hacia la escuela. En esta línea de trabajo se incluyen los estudios que han considerado el engagement como el nivel de "conectividad" que siente el estudiante con la escuela (Cornejo \& Redondo, 2001; Guerra et al., 2011; Marjoribanks, 1980), así como aquellos estudios que han incorporado el concepto de apego secundario para hablar de vínculos pedagógicos positivos (Mena et al., 2011).

c) Participación. La participación de los estudiantes en la toma de decisión y en el diseño de estrategias para prevenir la violencia escolar, ha mostrado ser un camino efectivo para reducir los niveles de violencia en la escuela (Flannery, 1997). Más allá de este argumento instrumental, la participación de los estudiantes es considerada un derecho (LLECE, 2008) que aumenta el compromiso y el nivel de agenciamiento de los estudiantes con sus procesos educativos. Siguiendo a Epstein (2001), dar voz a los estudiantes significa crear espacios de convivencia democrática. Hay evidencia a nivel internacional (Epstein, 2001) y en Chile (Montecinos, Sisto \& Ahu- 
mada, 2010) de una mayor participación y sentido de pertenencia de los distintos actores de la comunidad escolar en las escuelas orientadas a la mejora educativa.

\section{Escala de Clima Escolar}

La Escala de Clima Escolar reportada aquí es una adaptación del California School Climate Inventory propuesto por Khoury-Kassabri et al. (2004) como un cuestionario de autorreporte que permite una estimación fiable del clima escolar, considerando las tres dimensiones: normas claras, participación y apoyo de profesores, descritas anteriormente. Así, considera aspectos de participación en la organización escolar, de calidad de las relaciones entre pares estudiantes y de estos con los docentes y la aplicación de normas institucionales.

\section{Método}

\section{Participantes}

Participaron 4688 estudiantes de cuarto $(n=1$ $521 ; 32.4 \%)$, sexto $(n=1648 ; 35.2 \%)$ y octavo $(n$ $=1519 ; 32.4 \%$ ) año básico de escuelas urbanas con enseñanza básica en la región de Valparaíso (51\% mujeres). El procedimiento de muestreo fue aleatorio bietápico por estratos. Se utilizaron dos estratos: los niveles de concentración de estudiantes (menor a 300 estudiantes; entre 300 y 500 estudiantes; mayor a 500 estudiantes) y la proporción de estudiantes en los establecimientos según tipo de dependencia escolar (municipalizada, particular-subvencionada y particular-pagada). En la primera etapa, se seleccionaron aleatoriamente escuelas a partir del marco de muestreo según el estrato correspondiente. En la siguiente etapa, se seleccionó aleatoriamente un curso de cada uno de los tres niveles (cuarto, sexto y octavo) en las escuelas aleatoriamente seleccionadas. El procedimiento de muestreo significó la participación de 236 cursos en 81 escuelas con enseñanza básica en la región de Valparaíso.

\section{Instrumentos}

\section{Escala de Clima Escolar}

Se utilizó la Escala de Clima Escolar del Instrumento para Estudiantes elaborado por Khoury-Kassabri et al. ([2004]; Benbenishty \& Astor, 2005). Se trata de la adaptación de la versión para investigación del California School Climate and Safety Survey ([CSCSS]; Furlong, 1996). El Instrumento para Estudiantes contiene más de 100 preguntas referidas a distintas áreas, que conforman distintas escalas: victimización por pares y por el personal de la escuela, porte de armas en la escuela, conductas de riesgo, percepción y evaluación de violencia escolar y CE. Dado el interés de los proyectos de investigación en el cual se inserta en este estudio (FONDECYT № 1110859 y FONDEF-IDEA CA12I10243), nuestro propósito fue utilizar y validar la escala de CE.

La Escala de Clima Escolar utilizada por Benbenishty y Astor (2005) consta de 18 ítems con respuesta tipo Likert. Fue validada con una muestra de 7000 estudiantes de niveles de enseñanza 4 a 11 en Israel, después de un proceso de back-translation. La validación del instrumento en Israel y Taiwán mostró adecuadas propiedades psicométricas, siendo la fiabilidad más alta para las dimensiones de normas y políticas ( $\alpha$ entre 0.84 y 0.85 ) y apoyo de profesores ( $\alpha$ entre 0.86 y 0.88 ) que para la participación de estudiantes ( $\alpha$ entre 0.53 y 0.63) (Astor, Benbenishty, Zeira \&Vinokur, 2002; Chen \& Wei, 2011). Nueve ítems indagan la opinión de los estudiantes sobre las políticas y procedimientos de la escuela dirigidos a disminuir la violencia escolar (normas y políticas); tres ítems indagan los sentimiento de los estudiantes respecto de si ellos juegan un papel importante y activo en manejar la violencia en su escuela y seis ítems las relaciones de apoyo percibidas por parte de sus profesores.

Benbenishty y Astor (2005) encontraron que todos los ítems podrían ser explicados por un factor único $(\alpha=0.92)$, con un autovalor inicial de 9.2, donde los factores dos y tres tuvieron autovalores iniciales de 1.29 y 1.08 , respectivamente. Sin embargo, dada la fundamentación teórica que guía la generación del instrumento, decidieron trabajar 
con los ítems bajo la estructura tridimensional anteriormente descrita. En el presente estudio, se probó la estabilidad de esta solución tridimensional, así como la factibilidad de utilizar la escala como media unidimensional, dependiendo de los fines de la investigación. Para ello, se tradujo la escala publicada en inglés (Benbenishty \& Astor, 2005), siguiendo el método estándar de back-translation y ajustando algunos ítems a la jerga chilena.

\section{Procedimiento}

Los cuestionarios fueron aplicados en las escuelas durante el horario escolar, tras la autorización de los establecimientos y el consentimiento informado de los apoderados. Los datos fueron analizados utilizando el paquete estadístico SPSS 18.0. De un total de 4762 encuestas originalmente contestadas, se eliminaron 74 (1.5\%) que contenían más de 10\% de respuestas perdidas. El tratamiento de las respuestas perdidas se realizó mediante la media de los valores de las dimensiones originales. Las características psicométricas del instrumento fueron analizadas mediante alpha

\section{TABLA 2}

Ítems y saturaciones factoriales para la solución ortogonal VARIMAX de cuatro factores de clima escolar

\begin{tabular}{|c|c|c|c|c|}
\hline \multirow[b]{2}{*}{ Ítem } & \multicolumn{4}{|c|}{ Cargas factoriales } \\
\hline & $\begin{array}{c}1 \\
\text { AS }\end{array}$ & $\stackrel{2}{N}$ & $\begin{array}{l}3 \\
\mathrm{NV}\end{array}$ & $\begin{array}{l}4 \\
\mathrm{P}\end{array}$ \\
\hline $\begin{array}{l}\text { Cuando los estudiantes rompen las reglas, son tratados con firmeza pero de } \\
\text { manera justa. }\end{array}$ & 0.04 & 0.71 & 0.13 & 0.08 \\
\hline Mis profesores son justos. & 0.39 & 0.63 & 0.12 & 0.05 \\
\hline Obedecer las reglas en mi escuela tiene beneficios. & 0.17 & 0.58 & 0.11 & 0.18 \\
\hline Las reglas en mi escuela son justas. & 0.35 & 0.66 & 0.14 & 0.11 \\
\hline $\begin{array}{l}\text { Los profesores hacen un buen trabajo protegiendo a los estudiantes de los } \\
\text { revoltosos. }\end{array}$ & 0.42 & 0.37 & 0.12 & 0.29 \\
\hline $\begin{array}{l}\text { Cuando me quejo de alguien que me está haciendo daño, los profesores me } \\
\text { ayudan. }\end{array}$ & 0.54 & 0.3 & 0.27 & 0.13 \\
\hline En mi escuela hay reglas claras y conocidas contra la violencia. & 0.29 & 0.3 & 0.58 & 0.13 \\
\hline En mi escuela hay reglas claras y conocidas contra el acoso sexual. & 0.1 & 0.16 & 0.81 & 0.11 \\
\hline $\begin{array}{l}\text { Cuando los compañeros/as acosan sexualmente a otros compañeros/as, los } \\
\text { profesores los detienen. }\end{array}$ & 0.2 & 0.07 & 0.75 & 0.13 \\
\hline $\begin{array}{l}\text { En mi escuela, los estudiantes participan tomando decisiones importantes y } \\
\text { haciendo las reglas. }\end{array}$ & 0.14 & 0.13 & 0.03 & 0.78 \\
\hline $\begin{array}{l}\text { En mi escuela los estudiantes juegan un rol importante cuando se trata de } \\
\text { hacerse cargo de problemas de violencia }\end{array}$ & 0.09 & 0.09 & 0.19 & 0.74 \\
\hline $\begin{array}{l}\text { El personal de mi escuela se esfuerza en que los estudiantes participen en las } \\
\text { decisiones importantes. }\end{array}$ & 0.38 & 0.22 & 0.16 & 0.53 \\
\hline $\begin{array}{l}\text { Cuando los estudiantes tienen una emergencia (o un problema serio), un } \\
\text { adulto siempre está allí para ayudar. }\end{array}$ & 0.61 & 0.12 & 0.21 & 0.25 \\
\hline Mis profesores me respetan. & 0.68 & 0.17 & 0.23 & 0.03 \\
\hline Puedo confiar en la mayoría de los adultos en esta escuela. & 0.71 & 0.14 & 0.1 & 0.09 \\
\hline Mi relación con mis profesores es buena y cercana. & 0.73 & 0.19 & 0.02 & 0.09 \\
\hline Los profesores en esta escuela cuidan a los estudiantes. & 0.69 & 0.21 & 0.25 & 0.15 \\
\hline Me siento cómodo/a hablando con mis profesores cuando tengo un problema. & 0.72 & 0.16 & 0.05 & 0.17 \\
\hline Autovalor inicial & 6.5 & 1.29 & 1.1 & 1.04 \\
\hline$\%$ de varianza & 36.12 & 7.16 & 6.09 & 5.78 \\
\hline
\end{tabular}

$\mathrm{A}=$ apoyo social; $\mathrm{N}=$ normas; $\mathrm{NV}=$ normas contra violencia; $\mathrm{P}=$ participación Fuente: elaboración propia 
de Cronbach para el análisis de consistencia interna, análisis factorial exploratorio y confirmatorio para el análisis de validez de constructo, y correlaciones bivariadas para análisis de validez convergente.

\section{Resultados}

Validez de constructo

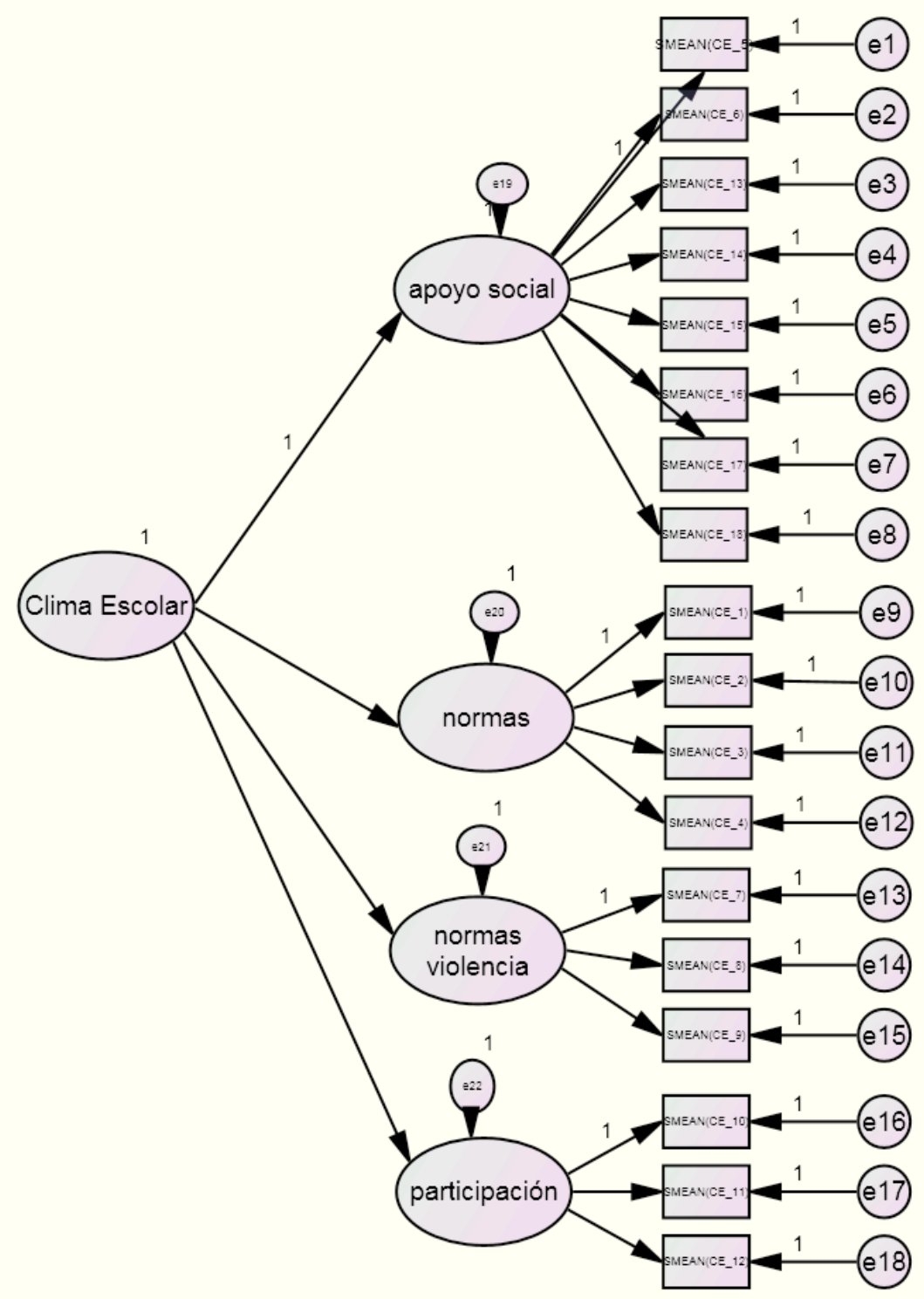

Figura 1. Modelo CFA de segundo orden propuesto para la Escala de Clima Escolar. Nota. Los constructos latentes se muestran en elipses, y las variables observadas en rectángulos.

Fuente: elaboración propia 
Para estudiar la validez de constructo se efectuó en primer lugar un análisis factorial exploratorio $\left(\mathrm{KMO}=0.943\right.$, Barlett $\left.\mathrm{X}^{2}=26675, p<0.001\right)$. La solución explicó el $54 \%$ de la varianza total. El primer factor explicó el 36\% de la varianza y presentó un autovalor inicial de 6.02, mientras que el segundo, tercer y cuarto factor tuvieron autovalores de 1.29, 1.1 y 1.04, respectivamente (Tabla 2). El análisis semántico permite deducir que se trata de las dimensiones de apoyo social, normas y políticas, normas contra violencia escolar y sexual y participación, respectivamente. Todos los ítems menos los ítems 5 y 6 corresponden a los ítems originalmente propuestos para la dimensión de normas. Los ítems 5 y 6 presentan una carga factorial dentro de la dimensión de apoyo social de profesores. Todos los ítems de participación se corresponden con los ítems originalmente propuestos para esta dimensión. A diferencia de la propuesta original, se observa que la dimensión de normas se separa en dos factores: normas y políticas de convivencia (Factor 2) y normas contra la violencia escolar y sexual (Factor 3).

En segundo lugar, se realizó un análisis factorial confirmatorio utilizando el módulo AMOS de SPSS. Se probó un modelo con las cuatro dimensiones como variables latentes, y los ítems con carga factorial en cada dimensión, según los resultados del análisis factorial exploratorio, como variables observadas. El modelo de cuatro dimensiones de primer orden mostró un buen ajuste Byrne, 2010. Incluirlo en la lista de referencias. la prueba de chi cuadrado - de ajuste mínimo del modelo- fue significativa $\left(X^{2}=1559.41, p<0.001\right)$, el indicador CFI (Comparative Fit Index) fue mayor a 0.9 $(\mathrm{CFI}=0.946)$ y el indicador RMSEA (Root Mean Square Error of Approximation) fue inferior a 0.5 (RMSEA $=0.049)$. Según Byrne (2010), estos parámetros indican un buen ajuste del modelo a la población general.

Se probó un segundo modelo de dos niveles, con las dimensiones latentes agrupadas bajo otra variable latente de segundo orden, CE (Figura 1). Los niveles de ajuste también fueron buenos $\left(\mathrm{X}^{2}\right.$ $=2065.30, p<0.001 ; \mathrm{CFI}=0.927$; RMSEA $=$ 0.056), aunque levemente inferior a la solución de primer orden, lo que es esperable por la parsimonia. Por último, para evaluar la posibilidad de utilizar la escala sin los ítems de acoso sexual, se probó un modelo de primer orden con las tres dimensiones originales: normas, apoyo social y participación. Los niveles de ajuste fueron nuevamente buenos para el modelo CFA de primer orden con tres factores $\left(\mathrm{X}^{2}=1027.39, p<0.001 ; \mathrm{CFI}=0.957\right.$; RMSEA $=$ 0.048) y levemente inferior para el modelo CFA de segundo orden con tres factores $\left(\mathrm{X}^{2}=1445.08, p<\right.$ 0.001; $\mathrm{CFI}=0.938$; RMSEA $=0.057)$. Esto indica que, en caso de que el investigador quisiera omitir los ítems de acoso sexual, el modelo de tres factores mantiene adecuadas propiedades psicométricas.

\section{Fiabilidad}

La consistencia interna de la escala total fue alta $(\alpha=0.89$; hombres $=0.888$; mujeres $=0.891)$. $\mathrm{La}$ Tabla 4 sintetiza los ítems originales para las tres dimensiones originalmente propuestas (normas, participación y apoyo social), así como los ítems para la solución de cuatro factores presentadas en este informe, junto a los respectivos valores de consistencia interna $(\alpha)$. Como se puede observar, si bien la estructura de tres dimensiones originalmente propuestas presenta un nivel de consistencia interna más estable, el nivel de consistencia interna de la solución de cuatro dimensiones es igualmente adecuada.

\section{Diferencias por género y tipo de escuela}

La Tabla 4 muestra las medias por género. Como se puede apreciar, ambos grupos perciben mayor apoyo social de profesores, que participación de ellos en la escuela. Las mujeres perciben un mayor apoyo social que los varones $\left(t\left(_{4682}\right)=3.073, p<\right.$ .01) y los varones una mayor presencia de normas de convivencia $\left.\left(t_{4682}\right)=1.983, p<.05\right)$.

Los análisis multivariantes indicaron que, en todas las dimensiones $\left(F_{2,4685}\right)=160.97$ para normas; 20.57 para normas contra violencia; 114.07 para participación y 178.62 para apoyo social, $p<$ 0.001 para todas las dimensiones), los estudiantes de cuarto básico reportan una mejor percepción del CE 
TABLA 3

Items de las dimensiones de la Escala de Clima Escolar

\begin{tabular}{|c|c|c|c|}
\hline & $\begin{array}{c}\text { Estructura original } \\
\text { (Astor et al., 2002; Benbenishty } \\
\text { \& Astor, 2005; Che \& Wei, 2011) }\end{array}$ & $\begin{array}{l}\text { Estructura original } \\
\text { (versión español) }\end{array}$ & $\begin{array}{l}\text { Estructura propuesta para } \\
\text { la versión en español }\end{array}$ \\
\hline Normas claras & $\begin{array}{c}1,2,3,4,5,6,7,8 \text { y } 9 \\
\alpha=0.84 \text { a } 0.85\end{array}$ & $\begin{array}{c}1,2,3,4,5,6,7,8 \text { y } 9 \\
\alpha=0.81\end{array}$ & $\begin{array}{l}1,2,3 \text { y } 4 \\
\alpha=0.69\end{array}$ \\
\hline Normas contra la violencia & -. & .. & $\begin{array}{c}7,8 \text { y } 9 \\
\alpha=0.68\end{array}$ \\
\hline Participación & $\begin{array}{c}10,11 \text { y } 12 \\
\alpha=0.53 \text { a } 0.63\end{array}$ & $\begin{array}{c}10,11 \text { y } 12 \\
\alpha=0.62\end{array}$ & $\begin{array}{c}10,11 \text { y } 12 \\
\alpha=0.62\end{array}$ \\
\hline Apoyo social & $\begin{array}{c}13,14,15,16,17 \text { y } 18 \\
\alpha=0.86 \text { a } 0.88\end{array}$ & $\begin{array}{c}13,14,15,16,17 \text { y } 18 \\
\alpha=0.84\end{array}$ & $\begin{array}{c}5,6,13,14,15,16,17 \text { y } 18 \\
\alpha=0.86\end{array}$ \\
\hline
\end{tabular}

Fuente: elaboración propia

en comparación a los estudiantes de sexto básico, los que a su vez presentan puntajes más altos que los estudiantes de octavo básico. No hay diferencias significativas entre escuelas estudiantes de escuelas públicas-municipales $(n=1596)$, particular-subvencionadas $(n=2955)$ y particular-pagadas $(n=137)$ $\left(F_{2,4685}\right)=1.82$ para normas; 2.24 para normas contra violencia; 1.86 para participación y 0.55 para apoyo social, $p>0.1$ para todas las dimensiones).

\section{Discusión}

Si bien los niveles de fiabilidad o consistencia interna son levemente inferiores a los reportados en Israel y Taiwán (Astor et al., 2002; Chen \& Wei, 2011) para las dimensiones de normas, esto se explica por el menor nivel de ítems por dimensión de la propuesta final. Los ítems 5 y 6 , a su vez, presentan cargas factoriales en el Factor 1 (apoyo social), lo que indica que, a lo menos para esta muestra de estudiantes chilenos, la existencia de normas de convivencia no necesariamente es lo mismo que la existencia de normas contra la violencia, sea ésta escolar o de acoso sexual al interior de la escuela.

En nuestro estudio, encontramos que la solución original de tres dimensiones, así como la de cuatro dimensiones, tienen adecuadas propiedades psicométricas. También lo tiene la solución de un factor de segundo orden que agrupa estas dimensiones en un nivel de jerarquía mayor. Para fines de investigación comparativa internacional y transcultural, se recomienda utilizar la escala bajo una estructura unidimensional o bajo la estructura tridimensional originalmente propuesta por Benbenishty y Astor (2005). En cambio, para fines de investigación a nivel local, resulta más informativo la solución de cuatro factores. A futuro, se sugiere estudiar con mayor profundidad la invarianza de los modelos según etapa del desarrollo evolutivo de los estudiantes.

\section{Referencias}

Aron, A. M., Milicic, N., \& Armijo, I. (2012). Clima social escolar: una escala de evaluación -Escala de

TABLA 4

Medias y desviaciones estándar en las dimensiones de clima escolar para hombres $(n=2295)$ y mujeres $(n=2393)$

\begin{tabular}{ccccccccc}
\hline & \multicolumn{2}{c}{ Normas } & \multicolumn{2}{c}{ Normas contra violencia } & \multicolumn{2}{c}{ Participación } & \multicolumn{2}{c}{ Apoyosocial } \\
\hline & $\mathrm{M}$ & $\mathrm{DE}$ & $\mathrm{M}$ & $\mathrm{DE}$ & $\mathrm{M}$ & $\mathrm{DE}$ & $\mathrm{M}$ & $\mathrm{DE}$ \\
\cline { 2 - 9 } Hombre & 3.99 & $(0.81)$ & 4.19 & $(0.86)$ & 3.65 & $(0.9)$ & 4.06 & $(0.76)$ \\
Mujer & 3.95 & $(0.81)$ & 4.23 & $(0.84)$ & 3.69 & $(0.88)$ & 4.13 & $(0.75)$ \\
\hline
\end{tabular}

Fuente: elaboración propia 
Clima Social Escolar, ECLIS-. Universitas Psychologica, 11(3), 803-813.

Ascorra, P., Arias, H., \& Graff, C. (2003). La escuela como contexto de contención social y afectiva. Revista Enfoques Educacionales, 5(1), 117-135.

Assael, J., \& Neumann, E. (1991). Clima Emocional en el Aula: un estudio etnográfico de las prácticas pedagógicas. Santiago: PIIE.

Astor, R. A., Benbenishty, R., Zeira, A., \& Vinokur, A. (2002). School climate, observed risky behaviors, and victimization as predictors of high school students' fear and judgments of school violence as a problem. Health Education $\mathbb{E}$ Behavior, 29(6), 716-736.

Benbenishty, R., \& Astor, R. (2005). School violence in context: Culture, neighborhood, family, school, and gender. Oxford: University Press.

Brand, S., Felner, R. D., Seitsinger, A., \& Hupkau, A. (2006, abril). Learning support indicators: School climate. Trabajo presentado en la Annual Meeting of the American Educational Research Association, San Francisco, EE. UU.

Bronfenbrenner, U., \& Morris, P. (2006). The bioecological model of human development. En R. M. Lerner \& W. Damon (Eds. de la serie) \& R. M. Lerner (Ed. del volumen), Handbook of child psychology: Vol. 1. Theoretical models of human development (6.a ed., pp. 793-828) New York: Wiley.

Byrne, B. M. (2013). Structural equation modeling with Amos. New York : Routledge.

California Department of Education (1998). The California Healthy Kids Survey. San Francisco: WestEd.

Carrasco, C. López, V. \& Estay, C. (2012). Análisis crítico de la ley de violencia escolar. Psicoperspectivas, 11(2), 31-55.

Chen, J., \& Wei, H. (2011). Student victimization by teachers in Taiwan: Prevalence and associations. Child Abuse Eु Neglect, 35(5), 382-390.

Cornejo, R., \& Redondo, J. (2001). El clima escolar percibido por alumnos de enseñanza media. Revista Última Década, 9(15), 11-52.

Elsaesser, C., Gorman-Smith, D., \& Henry, D. (2013). The role of the school environment in relational agression and victimization. Journal of Youth Adolescence, 42, 235-249.
Epstein, J. L. (2001). School, family, and community partnerships: Preparing educators and improving schools. Boulder, CO: Westview Press.

Flannery, R. (1997). Violence in America: Coping with drugs, distressed families, inadequate schooling, and acts of hate. New York: Continuum.

Furlong, M. J. (1996). Tools for assessing school violence. En S. Miller, J. Brodine \& T. Miller (Eds.), Safe by design: Planning for peaceful school communities (pp. 71-84). Seattle, WA: Committee for Children.

Goodenow, C. (1993). Classroom belonging among early adolescent students relationships to motivation and achievement. The Journal of Early Adolescence, 13(1), 21-43.

Furlong, M. J. (2005). California School Climate and Safety Survey. Recuperado de http://education.ucsb. edu/ schpsych/research.html

Guerra Vio, C., Castro Arancibia, L., \& Vargas Castro, J. (2011). Examen psicométrico del Cuestionario de Clima Social del Centro Escolar en estudiantes chilenos. Psicothema, 23(1), 140-145.

Haynes, N. M., Emmons, C. L., \& Comer, J. P. (1993). Elementary and Middle School Climate Survey. New Haven, CT: Yale University Child Study Center.

Jiménez, F. (2012). Violencia escolar en contextos educativos multiculturales: Una aproximación desde los modelos de gestión de la diversidad cultural. Psicoperspectivas, 11(2), 8-30.

Johnson, B. (2010). Exploring and explicating the distinctive features of educational organizations: Theories and theorizing. En W. K. Hoy \& M. DiPaola's (Eds.), Analyzing school context: Influences of principals and teachers in the service of students (pp. 1-38). Charlotte, NC: IAP.

Kelley, E. A. (1989). Improving school climate. Practitioner, 15(4), 1-5.

Khoury-Kassabri, M., Benbenishty, R., Astor, R. A., \& Zeira, A. (2004). The contributions of community, family, and school variables to student victimization. American Journal of Community Psychology, 34(3-4), 187-204.

López, V. \& Ascorra, P. (2012). Miradas a la violencia en el espacio social de la escuela: Editorial. Psicoperspectivas $11(2), 1-7$. 
López, V., Ascorra, P., Bilbao, A., Morales, M., Moya, I., \& Oyanedel, J. C. (2012). El ambiente escolar incide en los resultados PISA 2009: resultados de un estudio de diseño mixto. En Centro de Estudios MINEDUC (Ed.), Evidencias para políticas públicas en educación (pp. 49-94). Santiago de Chile: Ministerio de Educación.

López, V., Bilbao, M., \& Rodríguez, J. I. (2012). La sala de clases sí importa: incidencia del clima de aula sobre la percepción de intimidación y victimización entre escolares. Universitas Psychologica, 11(1), 91-101.

Marjoribanks, K. (1980). Schools, families and children's achievements. Studies in Educational Evaluation, 6(3), 253-264.

Marshall, M. (2005). Examining school climate: Deffning factors and educational inffluences Georgia: Center for Research on School safety, School Climate and Class Room Management, Georgia State University.

Mena, M. I., Becerra, S., \& Castro, P. (2011). Gestión de la convivencia escolar en Chile: problemáticas, anhelos y desafíos. En J. Catalán (Ed.), Psicología educacional: proponiendo rumbos, problemáticas y aportaciones (pp. 81-112).

Milicic, N., \& Aron, A. M. (2000). Climas sociales tóxicos y climas sociales nutritivos para el desarrollo personal en el contexto escolar. Psykhe, 9(2), 117-123.

Montecinos, C., Sisto, V., \& Ahumada, L. (2010). The construction of parents and teachers as agents for the improvement of municipal schools in Chile. Comparative Education, 46(4), 487-508.
Moos, R. H., Moos, B. S., \& Trickett, E. J. (1987). Escalas de clima social: familia, trabajo, instituciones penitenciarias, centro escolar. Madrid: TEA.

Moos, R. H. \& Trickett, E. J. (1974). Classroom environment scale manual. Palo Alto: Consulting Psychologist Press.

Orton, J. D., \& Weick, K. E. (1982). Loosely coupled systems: A reconceptualization. Academy of Management Review, 15(2), 203-223.

Swearer, S. M., Espelage, D. L., Koenig, B., Berry, B., Collins, A., \& Lembeck, P. (2012). A social-ecological model of bullying prevention and intervention in early adolescence. En S. R. Jimerson, A. B. Nickerson, M. J. Mayer \& M. J. Furlong (Eds.), The handbook of school violence and school safety: International research and practice (pp. 333-355). New York: Routledge.

Trianes, M. V., Blanca, M. J., de la Morena, L., Infante, L., \& Raya, S. (2006). Un cuestionario para evaluar el clima social del centro escolar. Psicothema, 18(2), 272-277.

Véliz-Burgos, A. (2012). Propiedades psicométricas de la escala de bienestar psicológico y su estructura factorial en universitarios chilenos. Psicoperspectivas, 11(2), 143-163.

Werblow, J., Robinson, Q. L., \& Duesbery, L. (2010). Regardless of school size, school climate matters: How dimensions of school climate affect student dropout rate. En W. K. Hoy \& M. DiPaola's (Eds.), Analyzing school contexts: Influences of principals and teachers in the service of students (pp. 191-208). Charlotte, NC: Information Age Publishing. 
